\title{
Points to consider for informed consent for genome/exome sequencing
}

\author{
ACMG Board of Directors
}

In its recently released report, "ACMG Recommendations for Reporting of Incidental Findings in Clinical Exome and Genome Sequencing," the American College of Medical Genetics and Genomics (ACMG) created a set of recommendations addressing incidental findings and a minimum list of conditions, genes, and variants that are recommended to be returned whenever clinical sequencing is performed. The ACMG recommended that, for the conditions on the list, the laboratory should return the incidental findings to the doctor ordering the sequencing, and those doctors should manage this information with the patient in the context of that patient's clinical presentation and family history. This document, "Points to Consider for Informed Consent for Genome/Exome Sequencing," focuses on the need for and content of the informed consent that should be obtained before clinical applications of genome sequencing and exome sequencing (GS/ES) for germ-line testing.

GS/ES are rapidly transitioning into clinical practice. Initial applications include testing based on clinical indications that permit the targeting of specific and multiple genes/variants. Consent issues for such applications differ little from those already in use in genetics. However, many unique issues arise when testing platforms are used that provide information that extends beyond the specific genes of interest. ${ }^{1}$ These include issues of informed consent that are the focus of this "points to consider" document.

The types of information derived from genome sequencing will be both health (current and future) and non-health related. In addition to information specific to clinical indications for testing on a GS/ES platform, they include: gene-variant carrier status that may have implications for reproductive decision making; information about disease susceptibility or predisposition; information about ancestry, which is currently mostly informational but that may in time have clinical utility; and diagnosis of unsuspected disorders. Some phenotypes may allow narrow targeting, and others (e.g., intellectual disability or autism spectrum disorder) may leave the majority of the exome open for testing. Current technologies are being applied in both the postnatal and prenatal settings as well as to somatic and germ-line conditions. In some cases, incidental findings can be as important to a family as they are to the individual.
The following are recommendations regarding the informed consent that should be obtained before clinical applications of GS/ES for germ-line testing. Particular focus is placed on situations in which the laboratory and physician may be presented with information apparently unrelated to genes known to be associated with the phenotype that led to testing. These points reiterate some prior ACMG positions on this topic.

1. Before initiating GS/ES, counseling should be performed by a medical geneticist or an affiliated genetic counselor and should include written documentation of consent from the patient.

2. Incidental/secondary findings revealed in either children or adults may have high clinical significance for which interventions exist to prevent or ameliorate disease severity. Patients should be informed of this possibility as a part of the informed consent process.

3. Pretest counseling should include a discussion of the expected outcomes of testing, the likelihood and type of incidental results that may be generated, and the types of results that will or will not be returned. Patients should know if and what type of incidental findings may be returned to their referring physician by the laboratory performing the test.

4. Patients should be counseled regarding the potential benefits and risks of GS/ES, the limitations of such testing, potential implications for family members, and alternatives to such testing.

5. GS/ES is not recommended before the legal age of majority except for:

a. Phenotype-driven clinical diagnostic uses;

b. Circumstances in which early monitoring or interventions are available and effective; or

c. Institutional review board-approved research.

6. As part of the pretest counseling, a clear distinction should be made between clinical and research-based testing.

7. Patients should be informed as to whether individually identifiable results may be provided to databases, and they should be permitted to opt out of such disclosure. 
8. Patients should be informed of policies regarding re-contact of referring physicians as new knowledge is gained about the significance of particular results.

These points to consider were designed primarily as an educational resource for clinical geneticists and genetic counselors to help them provide quality clinical genetic services. Adherence to these points to consider is completely voluntary and does not necessarily ensure a successful clinical outcome. These points to consider should not be considered inclusive of all proper procedures or exclusive of other procedures that are reasonably directed to obtaining the same results. In determining the propriety of any specific procedure, the clinician should apply his or her own professional judgment to the specific clinical circumstances presented by the individual patient or specimen.

Clinicians are encouraged to document the reasons for the use of a particular procedure, whether or not it is in conformance with these points to consider. Clinicians also are advised to take notice of the date this guideline was adopted and to consider other medical and scientific information that becomes available after that date.

\section{REFERENCE}

1. ACMG Board of Directors. Points to consider in the clinical application of wholegenome sequencing. Genet Med 2012;14:759-761. 